\title{
Searching for the best evidence. Part 1: where to look
}

Clinicians are continually challenged to keep up with the rapidly growing and changing information base relevant to their areas of practice. They must not only locate relevant information but must also assess its quality, asking themselves do I, or should I, believe this information? Increasingly, they must deal with conflicting information. For example, 1 study may report that treatment $A$ was better than treatment $B$, but another study may report just the opposite. This is the first of 2 editorials on searching for the best evidence. It will describe the 5 main types of information sources that can be used to meet the information needs of nurses, with examples of the types of sources that are best suited to addressing different kinds of questions. Part 2 of this editorial will focus on techniques for the efficient retrieval of high quality information from an important information source for nurses-that is, electronic bibliographic databases, specifically CINAHL and Medline.

For nurses, most information needs can be met using 5 types of sources: textbooks, journals, online bibliographic databases, products that distill or consolidate research, and the internet. Each will be discussed in turn below.

\section{Textbooks}

Textbooks provide information for 2 types of needs. Whether the texts are traditional or on CD ROM disks, books are invaluable for addressing specific "stable" information needs - that is, facts that do not often change such as gross anatomy, basic principles and mechanisms, and specific disease characteristics. If they are up to date, texts can also provide summaries of new or complex topics, or put an issue in the context of other related areas of knowledge. Examples of the type of questions that can be answered using textbooks are: what are the developmental milestones for 2 year old children? and what is the incubation period for chickenpox?

Many health professionals feel that they need 1 or 2 standard general textbooks and several specialty texts to cover their specific area of expertise (eg, neurology or paediatrics). It is important to remember, however, that most textbooks are at least 2 years old when they are published because of the time required for preparation, editing, and production. Furthermore, authors often do not provide references to back up their claims and enrich their chapters.

\section{Journals}

Journals provide a forum for clinicians to share learning and experiences in their practice and profession. Most importantly, however, they provide a mechanism to keep readers up to date with current research on a variety of topics.

More than 400 nursing journals are included in Ulrich's International Periodicals Directory. ${ }^{1}$ When selecting which of these journals to read or to subscribe to (for the purpose of keeping up to date with new research), nurses might consider the following questions:

- Is the journal peer reviewed? (ie, are articles submitted for publication reviewed and evaluated by experts in the field?)
- Is the journal local, national, or international in scope?

- Does it include research articles that report on the methods and findings of qualitative or quantitative research studies or is it more general in nature, reporting on professional news, personal experiences of clinicians, and general discussions about current clinical issues?

Although nursing journals report on advances in research and practice that are relevant to nurses, important advances in any area of health care are usually published in the major general medical journals-BMJ, New England Journal of Medicine, Lancet, and JAMA. The concentration of important advances in these journals is because of tradition, prestige, and large circulation numbers. Many of these advances, however, have important implications for healthcare professions other than medicine. It is therefore important for nurses to consider these medical journals when deciding which journals they will read.

As an information source, journals have some limitations. One of the most obvious is referred to as "publication bias". It has been shown that research studies that report positive findings (ie, that an intervention had beneficial or harmful effects for patients) are more likely to be published or to be published more quickly than studies that report no differences. ${ }^{2}$ Therefore, studies which find that an intervention had no effect on patient outcomes are probably underrepresented in the published literature. Nurses may also encounter barriers to accessing relevant journals, such as the high cost of personal subscriptions and the unavailability of journals in some clinical settings.

The type of questions that can be answered by reading current issues of journals usually cover broad topic areas. For example, are there any new advances in the treatment of patients with diabetes mellitus? and is there new information that can help me to better understand how my patients experience their illnesses and their care? Reading current issues of journals is not, however, an efficient way of finding information on specific questions that may come up in everyday clinical practice. Alternative sources such as online bibliographic databases are better suited to answering these types of questions.

\section{Bibliographic databases}

Several large bibliographic databases provide nurses with access to citations (some with abstracts) for most original studies and reviews published in the healthcare literature. These databases include CINAHL (a general database for nurses and other allied health professionals from the US), Medline (a large general purpose database from the US), and Embase (a general purpose database from Excerpta Medica in the Netherlands). All include citations and the authors' abstracts, but the full texts of articles are not available electronically from these databases.

CINAHL (Cumulated Index to Nursing and Allied Health Literature) is produced by CINAHL Information Systems (www.cinahl.com/). The database has roots going back to the 1940 s and includes many years of articles indexed on 3 " x 5" 
filing cards. The computer version started in 1982 and includes citations to $>250000$ articles from $>650$ English language journals. Content is concentrated on nursing, physiotherapy, occupational therapy, emergency care, and alternative treatments. In addition to citations and abstracts, many of the entries include an extensive bibliography of related articles.

Medline is produced by the US National Library of Medicine. It includes $>8$ million citations to publications going back to 1966, with articles indexed from $>3500$ journals. Medline provides coverage of all areas of health care, especially research.

A useful paper by Burnham and Shearer provides a description and brief history of each of CINAHL, Medline, and Embase, as well as the results of nursing based searches in each of the 3 databases for specific clinical topics. ${ }^{3}$ Based on their results and our searching experience, we suggest using CINAHL and Medline for everyday search needs, and adding an Embase search when it is important to retrieve almost everything on a topic.

Different methods exist for accessing each database, and every system has its own searching style and entry mechanisms. In this editorial, we present only general searching techniques. More specific searching of CINAHL and Medline will be discussed in part 2 of this editorial.

When you search any large bibliographic database, you can do so using the terms and phrases that the authors have used in their titles and abstracts (often called textwords) or you can search using the index terms that the indexers have assigned to each article as it is prepared for entry into the database (eg, in Medline these are called Medical Subject Headings or MeSH terms). Each type of searching has strengths and weaknesses. Searching using terms from the title and abstract (textword searching) is often most effective for retrieving information on new ideas. For example, textword searching would probably be the best approach when looking for information on the effects of household smoking-a relatively new topic of study. It is also useful when trying to locate a specific study such as the Nurses' Health Study or the DCCT (Diabetes Control and Complications Trial). A textword approach would not be as effective when searching for information on a topic that has many different names or terms because the searcher would need to identify and include all of these terms when searching. For example, decubitus ulcers are known as bed sore, bed sores, bedsore, bedsores, pressure ulcer, pressure ulcers, pressure sore, pressure sores, and may also be referred to using terms such as "tissue viability". In this instance, searching using the database index terms (pressure ulcer for CINAHL and decubitus ulcer for Medline) would be more efficient.

Because these databases are large, retrievals based on single search terms are often not effective: CINAHL has 286 articles on elder abuse, 427 on handwashing, and 1653 on pressure ulcers and Medline has 483, 1042, and 4068 articles on the same topics. To reduce the number of retrievals and to allow for better refinement of questions, the Boolean algebra AND operator is used. By ANDing 2 or more concepts together, a searcher stipulates that only citations with both concepts are retrieved. In the handwashing example, by ANDing in the term "surgical wound infection" (ie, handwashing AND surgical wound infection), CINAHL retrieves 12 citations that are indexed for both handwashing and surgical wound infection and the most current subset of Medline (1995-8) retrieves 8 citations. The Boolean OR function can also be used to combine search concepts. ORs combine "like" ideas-for example, if you are interested in using either amoxicillin OR nasal decongestants for treating acute sinusitis, CINAHL has 4 articles and the current Medline database has 23 articles. Many complain that the use of ANDs and ORs in searching is counterintuitive. An easy way to remember their use is to consider a restaurant patron's request at ordering time and use the opposite approach-to a waitress, "peas and carrots" means both, and "peas or carrots" means only one-to a searcher, it is the opposite.

\section{Sources of distilled and consolidated information} DISTILLED INFORMATION SOURCES

Despite the peer review process, not all research studies published in journals are methodologically sound, and some may be more sound than others. The assessment of the quality of research studies (often called critical appraisal) will be the topic of future editorials. For now, we will focus on information sources which provide "distilled" information-that is, some of the critical appraisal work has already been done for the reader and only a "distillation" of the best studies is presented.

The evidence-based journal series (Evidence-Based Nursing, Evidence-Based Medicine, and Evidence-Based Mental Health) and ACP Journal Club are examples of distilled information sources. Research articles of the highest methodological quality are identified by searching the current international healthcare literature. Structured abstracts are prepared for selected articles (up to 24 articles/issue) on clinically relevant topics, and each abstract is accompanied by a commentary written by a clinician which links the study findings to clinical practice. Each abstract and accompanying commentary fits on 1 page.

While Evidence-Based Nursing focuses on nursing related research, ACP Journal Club, now in its 8th year of publication, is directed at internists; Evidence-Based Medicine, published for 3 years, is for general practitioners and others interested in a broad coverage of important advances in medicine; and Evidence-Based Mental Health, which published its first issue in February 1998, focuses on topics of interest to psychiatrists, psychologists, and other mental health professionals.

Regular reading of evidence-based abstract journals can address the general types of information needs discussed in the section on journals. The advantages are that only high quality studies are included and key information is provided in a concise format. Readers therefore save time on 2 fronts-they do not have to do the work of separating the "wheat" from the "chaff", and they do not have to read multiple pages of text to find the "bottom line".

Similarly, although the everyday information needs of nurses (ie, finding answers to specific clinical questions) can be addressed by searching large bibliographic databases, this requires the user to sift through numerous citations to find a few high quality relevant citations that answer their questions. Best Evidence is a distilled version of a bibliographic database (available on CD ROM) which currently includes all abstracts and commentaries that appear in ACP Journal Club and Evidence-Based Medicine, as well as citations to other high quality articles that were not selected for abstraction. In 1999, Best Evidence will expand to include material from EvidenceBased Nursing and Evidence-Based Mental Health.

The time saving advantages of Best Evidence are similar to those previously described for evidence-based abstract journals. The database is small ( $<2000$ articles) and easy and quick to search using simple textword searching. For example, the search term "hormone replacement therapy" retrieves 24 original studies and systematic review articles which include information on hormone replacement therapy in the context of stroke, breast cancer, cardiovascular disease, and fractures. 
CONSOLIDATED INFORMATION SOURCES

When searching for information on a specific topic, you will often find conflicting data from different studies on the same topic. Differences in results may occur because studies use different designs, methods, and outcomes. One specific type of study, the systematic review, attempts to reconcile these differences by synthesising the findings of multiple primary studies on the same topic. Systematic reviews differ from traditional narrative reviews in that they focus on a specific clinical question, use comprehensive sources and explicit search strategies to identify primary studies, select articles based on predetermined criteria, and critically appraise the selected studies. If judged to be sufficiently similar, the results of multiple studies may be combined using statistical methods. This type of systematic review is called a meta-analysis. ${ }^{4}$

The Cochrane Collaboration is an international network of healthcare professionals and lay people committed to producing and maintaining systematic reviews of the effects of healthcare interventions. The collected systematic reviews and citations of controlled trials and randomised controlled trials are published in the Cochrane Library (available on CD ROM or on the internet at http://www.cochrane.co.uk/for a fee). The Library includes 4 databases: (1) The Cochrane Database of Systematic Reviews contains the full text of $>300$ systematic reviews and protocols for $>300$ planned or ongoing systematic reviews. Systematic reviews have been done on many nursing related topics including adherence to medications, breast feeding, care giver support and postpartum depression, doctor-nurse collaboration, effects of limited asthma education, stroke units, support during childbirth, and treating scabies; (2) The Database of Abstracts of Reviews of Effectiveness (DARE) is produced by the UK NHS Centre for Reviews and Dissemination at the University of York and contains $>1500$ records of high quality systematic reviews published internationally. Numerous databases including CINAHL are regularly searched to identify high quality reviews, and each review is appraised by a trained reviewer using established quality criteria. In the Cochrane Library, DARE is updated quarterly; an internet version (http://nhscrd.york.ac.uk/welcome.html) is updated more frequently; (3) The Cochrane Review Methodology Database comprises references to articles on the theory and methods of systematic reviews and on critical appraisal; (4) The Cochrane Controlled Trials Register is a database of citations of trials and has $>150000$ entries.

\section{The internet}

The internet has brought both blessings and curses for nurses, as for others. It has provided health professionals and patients access to many valuable information sources. Unfortunately, because of the speed at which the internet is expanding and the lack of quality control of information that is published, these sources are often hidden in a morass of information that is of questionable, or at least uncertain, quality. Research about evaluating the quality of information found on internet sites is in its early stages. A systematic review by Jadad and Gagliardi identified 47 rating schemes for evaluating the quality of internet sites which provide health information, and all were incompletely developed and gave no information on test reliability and validity. ${ }^{5}$ Given this, it is important for nurses to judge the quality of information found on a given internet site using criteria based on common sense (eg, consider the source), checking it out with a colleague, and applying some of the principles of critical appraisal and evidence-based practice.
Nurses can search the internet to meet a variety of information needs. For example, they can help patients with newly diagnosed life threatening diseases to identify internet sites that provide accurate disease specific information from a professional perspective, in a form that is understandable from a patient perspective; online support groups; and electronic mailing lists. ${ }^{5}$ This provides an excellent opportunity for patients to become more involved in decision making on their own health care. Nurses can access some of the bibliographic databases discussed in the previous section at no cost. They can also learn about new topics by accessing internet tutorial sites published by reputable universities and organisations. The following box includes some examples of internet sites for some of the information needs previously discussed.

Examples of potentially useful internet sites

\section{Disease specific site}

Johns Hopkins AIDS service (www.hopkins-aids.edu/ default.htm) includes information on publications, treatment, managed care, epidemiology, and resources as well as a patient forum (where patient questions are answered by clinical experts) and an educational site (presentations of case rounds and a knowledge test).

\section{CINAHL and Medline access}

Dr Felix's internet page (195.152.9.7:80/drfelix) provides a list of free Medline services and a list of online free tutorials for CINAHL. No free access to CINAHL is currently available.

Pubmed and Internet Grateful Med (www.nlm.nih.gov/ databases/freemedl.html) are 2 new free services from the US National Library of Medicine which provide effective, powerful, and easy to use searching.

\section{Evidence-based practice}

Netting the evidence (www.shef.ac.uk/uni/academic/R-Z/ scharr/ir/netting.html) includes many references and sources related to evidence-based practice.

Evidence Based Nursing site at the University of York (www.york.ac.uk/depts/hstd/centres/evidence/ev-intro.htm)

\section{Summary}

Nurses need information frequently. Different needs are best met using specific sources or combinations of sources. Textbooks, journals, bibliographic databases, products that distill or consolidate research, and the internet each have their place in providing answers to information needs. A proactive approach to these sources, a decent computer, an internet connection and a small personal library can provide practising nurses with adequate information access at a reasonable cost in terms of money, time, and energy.

K ANN MCKIBBON, MLS

Health Information Research Unit, SUSAN MARKS, BA, BEd

McMaster University,

Hamilton, Ontario, Canada

1 Ulrich's international periodicals directory. New York: Bowker, 1996.

2 Stern JM, Simes RJ. Publication bias: evidence of delayed publication in a cohort study of clinical research projects. BMJ 1997;315:640-5.

3 Burnham J, Shearer B. Comparison of CINAHL, EMBASE, and MEDLINE databases for the nurse researcher. Med Ref Serv $Q$ 1993;12:45-57.

4 Mulrow C, Cook D, editors. Systematic reviews: synthesis of best evidence for health care decisions. Philadelphia: American College of Physicians, 1998.

5 Jadad AR, Gagliardi A. Rating health information on the internet: navigating to knowledge or Babel? JAMA 1998;279:611-4. 